\title{
The Temperature Decomposition Method for Simulating Periodic Thermal Flows
}

\author{
Junfeng Zhang \\ Professor in Mechanical Engineering \\ Bharti School of Engineering, Laurentian University \\ Sudbury, ON, Canada \\ jzhang@laurentian.ca
}

\begin{abstract}
Thermal flows through periodic structures are often encountered in many natural processes and industrial applications. By taking advantage of the similarity relationships of flow and thermal fields in periodic modules, computer simulations can be performed over a one-module domain, and the results can be applied to individual modules. Despite the computational attractiveness, this approach had been limited to systems with relatively simple boundary situations: either the temperature or heat flux can be specified over the solid surfaces. To address this concern, recently we have developed a temperature decomposition method that can work with more general boundary situations, such as systems with non-uniform wall temperature, mixed temperature and heat flux condition, convective boundary condition, and conjugate heat transfer.

In this method, the regular temperature is split into two components, namely the transient and equilibrium parts: the transient part decays with the flow and the regular temperature approaches the equilibrium part gradually. The two components can be solved independently under similar governing equations but different wall and inlet/outlet boundary conditions. The regular temperature can then be quickly obtained by adding them together according to the transient coefficients of individual periodic modules. The algorithm and implementation will be described, and example simulations are also presented to demonstrate the capacity and usefulness of this method for future simulations of thermal periodic flows.
\end{abstract}

